\title{
Procuras não urgentes a um pronto-socorro hospitalar pediátrico: perspectivas dos usuários
}

\author{
Nonurgent visits to a pediatric hospital emergency \\ department: users' perspectives
}

Tatiana Scheuer Becker ${ }^{1}$, Débora Carla Chong e Silva²

\begin{abstract}
1. Graduação em medicina pela Universidade Federal de Santa Catarina (UFSC). Pediatra pela Universidade Federal do Paraná (UFPR). Mestre em Saúde da Criança e do Adolescente pela Universidade Federal do Paraná (UFPR). ORCID: https://orcid.org/0000-0003-0583-9211

2. Doutora em Saúde da Criança e do Adolescente pela Universidade Federal do Paraná (UFPR). Docente do departamento de pediatria do Hospital de Clínicas da Universidade Federal do Paraná (UFPR) e da Pontifícia Universidade Católica do Paraná (PUC-PR). Supervisora do programa de residência médica em pediatria do Hospital de Clínicas da Universidade Federal do Paraná (UFPR).ORCID: https://orcid. org/0000-0002-7385-4598
\end{abstract}

CONTATO: Tatiana Scheuer Becker | Departamento de Pediatria Hospital de Clínicas da Universidade Federal do Paraná | Rua General Carneiro, 181 | CEP 80060-900 | Curitiba | Paraná | Telefone: (41) 99585-6430 | E-mail: tatianascheuerbecker@gmail.com

COMO CITAR: Becker TS, Silva DCC. Procuras não urgentes a um pronto-socorro hospitalar pediátrico: perspectivas dos usuários. R. Saúde Públ. Paraná. 2020 Jul;3(1):62-74.

(c)
(c) In

RESUMO Causas não urgentes são frequentes entre os pacientes nos prontos-socorros (PS) pediátricos, com consequências aos pacientes, aos profissionais e ao sistema. Este estudo objetiva descrever as procuras não urgentes a um PS de hospital de referência pediátrico, sendo um estudo observacional descritivo. Dos 168 participantes, 63\% haviam procurado previamente outros serviços de saúde. O principal motivo citado como razão de procura foi "ter o hospital como referência de resolutividade e confiança”. A maioria (68\%) dos entrevistados classificaram os quadros 
como urgências. Enquanto 63\% disseram não ter recebido orientações sobre sinais de alarme na criança, 83\% não haviam recebido informações sobre diferenças entre os níveis de atenção em saúde. Assim, foi demonstrada que a compreensão das razões de procura ao PS deve ser avaliada conjuntamente ao contexto social, de educação e de acesso à saúde dos usuários.

PALAVRAS-CHAVE: Serviços Médicos de Emergência. Medicina de Emergência Pediátrica. Pronto-Socorro.

\begin{abstract}
Nonurgent causes have been frequent in pediatric emergency departments (ED), leading to consequences for patients, professionals and the system. This study aimed to describe nonurgent pediatric visits to an ED of a reference pediatric hospital, as an observational descriptive study. Of 168 participants, $63 \%$ had sought prior care before going to the ED. The main reason cited for the search of the ED was "to have the hospital as a reference of problem-solving capacity and trust". Most caregivers (68\%) assessed their child's current condition as an urgency. While $63 \%$ reported not having received information about alarming symptoms in children, $83 \%$ did not receive information about the differences among levels of health care. The results showed that understanding the reasons to ED search should be analyzed in conjunction with the users' social,educational, and of health system access context.
\end{abstract}

KEYWORDS: Pediatric emergency medicine. Emergency medical services. Emergency department.

\title{
INTRODUÇÃO
}

$\mathbf{0}$ s prontos-socorros (PS) são locais destinados à assistência de usuários em situação de saúde de urgência e emergência, com estrutura e profissionais especializados para esse fim, podendo ser serviços hospitalares ou não hospitalares'. Entretanto, estudos demonstram que em média $50 \%$ do total de consultas nos PS na população pediátrica são casos classificados como não urgentes, problemas de saúde que poderiam receber tratamento ambulatorial2-5. Em Maceió, um estudo em uma unidade de emergência apontou que apenas 16,8\% das crianças e adolescentes atendidos em 1998, 2001 e 2004 apresentavam agravos adequados à complexidade oferecida pelo serviço ${ }^{6}$. Já em PS de hospitais de Recife, outro estudo revelou que apenas $15,2 \%$ dos casos que motivaram a demanda infantil eram compatíveis com o potencial tecnológico das unidades ${ }^{2}$.

A ocorrência da procura direta aos hospitais por parte dos usuários para atendimentos não urgentes sobrecarrega esses serviços, com repercussão aos profissionais da saúde e preocupação aos gestores do sistema ${ }^{6}$. Algumas consequências que ocorrem são tempo de espera prolongado, gerando insatisfação dos acompanhantes, das crianças e também da equipe médica que trabalha sobrecarregada7-8; descontinuidade dos cuidados de saúde; aumento de custos para todo o sistema, entre outros. 
Além disso, a visão da população sobre casos de urgência e emergência parece ser baseada em múltiplos fatores subjetivos, culturais e sociais, junto às vivências pessoais. Embora haja definições técnicas para urgência e emergência, a população usuária possui definições próprias que acaba por gerar a procura por esse serviço ${ }^{6}$. Assim, parece haver uma divergência entre o que é urgência para a mãe ou acompanhante e o que é para o profissional de saúde e gestores.

Outro ponto importante para análise da situação desses pacientes em situação não urgente é a análise da forma de estruturação e de assistência do Sistema Único de Saúde (SUS). Dificuldades de acesso aos serviços, falta de médicos nas unidades de saúde, tempo prolongado de espera para consultas e deficiências nas estruturas das unidades ${ }^{10}$, são algumas deficiências que ocorrem na atenção primária e secundária. Dessa forma, o PS torna-se uma porta de entrada atrativa aos usuários, pois possui funcionamento 24 horas, profissionais especializados e exames complementares nas próprias instituiç̧ões"1.

Alguns dos motivos da utilização dos PS pediátricos por causas não urgentes já foram destacados em estudos internacionais, como vantagens ao ser atendido nesses locais (rapidez, solicitação de exames), visão como centros mais especializados para consulta, insatisfação com os níveis de atenção primária e grande preocupação com o estado de saúde das crianças 5.10.12. Porém, ainda há poucos estudos neste sentido, especialmente na realidade brasileira, na qual os estudos demonstram fatores demográficos e epidemiológicos dos pacientes e, raros, são os que detalham as razões referidas pelos acompanhantes para levar os pacientes aos serviços de PS, quando em quadros de saúde não urgentes².13.

Diante da relevância do tema como questão de saúde pública, da especificidade da organização de saúde no contexto brasileiro, das diferenças sociais e culturais existentes entre os contextos dos estudos apresentados e dos poucos estudos nacionais, faz-se necessário buscar dados relativos à realidade nacional motivados pelos resultados expressivos encontrados em âmbito internacional. Este trabalho tem como principal objetivo descrever as procuras não urgentes a um PS de hospital de referência pediátrico, demonstrando as principais razões para a escolha desse local, a trajetória prévia dos pacientes a outros serviços de saúde e descrever o conhecimento dos acompanhantes sobre temas em saúde.

\section{MÉTODO}

Trata-se de uma pesquisa observacional descritiva com coleta de dados prospectiva, em que se visou descrever a população e as frequências das ocorrências. A coleta de dados foi realizada de setembro a novembro de 2018 no pronto-socorro SUS de um hospital pediátrico de referência em Curitiba. Também foi realizado o projeto piloto do estudo no mesmo local, em agosto de 2018, que comprovou a adequabilidade para a continuação da pesquisa.

Os sujeitos deste estudo foram os acompanhantes de crianças que foram triadas como casos não urgentes, por classificação de risco adotada pelo hospital, que frequentaram o PS de um hospital exclusivamente pediátrico da rede pública, de referência para Curitiba e região. Foram critérios de inclusão: acompanhantes de crianças e adolescentes de 0 a 18 anos atendidas no PS, cuja classificação de risco tenha apontado as procuras como de baixo risco de gravidade/não urgências e que tenham consentido a participação pela assinatura do Termo de Consentimento Livre e Esclarecido, e como exclusão: acompanhantes de crianças que haviam sido internadas recentemente, que representavam retornos para reavaliação médica do quadro de saúde prévio e quando os dados coletados foram incompletos.

A partir da população de estudo, foi obtida uma amostra não probabilística de conveniência sistematizada. 
A amostragem por conveniência é adequada e frequentemente utilizada para estudos exploratórios para geração de ideias e hipóteses. Nela, o pesquisador seleciona os elementos a que tem acesso, admitindo que estes podem representar um universo ${ }^{14-16}$. A pesquisadora foi a única pessoa que aplicou o instrumento de coleta e que esteve presente no local. A coleta foi realizada em sessões, de modo que contemplasse os turnos matutino, vespertino e noturno pelo menos uma vez para todos os dias da semana. Sua realização foi organizada de uma maneira viável aos meios disponiveis. Nos horários em que a pesquisadora esteve presente. selecionou a primeira criança/adolescente que havia sido classificada como não urgente pela classificação de risco adotada pela instituição. A partir daí, de acordo com o término de cada entrevista, uma criança a cada 15 minutos não sequencialmente à triagem, pois esta ocorria mais rapidamente do que o processo de aplicação das entrevistas. A pesquisadora coletou dados por aproximadamente 1 a 2 horas em cada turno.

O instrumento de coleta de dados foi elaborado a partir de extensa revisão da literatura, baseando-se nas principais variáveis abordadas dos estudos mais relevantes da área ${ }^{5.911}$. Continha perguntas de múltipla escolha e com respostas diretas, relacionadas às variáveis demográficas, variáveis relacionadas à procura pelo PS e variáveis relacionadas ao conhecimento de saúde e acompanhamento de saúde de rotina, sendo que a pesquisadora foi responsável pela aplicação das perguntas, com duração aproximada de 5 a 10 minutos cada.

A amostra foi calculada baseada na fórmula do cálculo do tamanho da amostra considerando a amostragem das proporções. Assim que testes estatísticos demonstraram diferenças estatisticamente significativas entre as variáveis com o número suficiente, verificando-se que a ampliação do número de participantes não alterava os resultados, optou-se por encerrar o procedimento de coleta de dados. As medidas de tendência central e de dispersão estão expressas em médias e desvio-padrão para as variáveis contínuas de distribuição simétrica e em medianas, valores mínimo e máximo, para as de distribuição assimétrica. As variáveis qualitativas foram expressas por meio de medidas de frequência absoluta e relativa. Os dados obtidos foram organizados em forma descritiva e por meio de tabelas de frequências.

O artigo é baseado na dissertação - Diagnóstico situacional das procuras não urgentes a um prontosocorro hospitalar pediátrico: perspectivas dos usuários, apresentada ao Programa de Pós-Graduação em Saúde da Criança e do Adolescente da Universidade Federal do Paraná (UFPR), em 2019. A pesquisa foi aprovada pelos Comitês de Ética das duas instituições envolvidas, Hospital de Clínicas da UFPR em 22/06/2017 e Hospital Pequeno Príncipe (HPP) em 02/08/2017, sob os respectivos números:2.131.678 e 2.197.999.

\section{RESULTADOS}

Foram realizadas 187 entrevistas no total, sendo que 17 casos constituíram a amostra do estudo piloto. Excluindo-se as coletas do estudo piloto, 170 acompanhantes responderam e em dois casos os dados não foram suficientes, totalizando 168 pacientes na amostra final do estudo. Nos períodos matutinos foram realizadas 45 coletas, 68 nos vespertinos e 55 nos noturnos. Dentre todos os atendimentos realizados no PS durante o período de coleta de dados (setembro a novembro de 2018), ocorreu média mensal de 67\% de atendimentos não urgentes.

A maioria ( $88 \%, n=148)$ dos acompanhantes eram mães, as quais também representaram o grupo que convivia mais tempo com as crianças $(78 \%, n=132)$. A média de idade dos acompanhantes foi de 31,4 anos. A tabela 1 demonstra as características demográficas dos pacientes. Noventa (53\%) eram do sexo masculino, com média de idade de 3 anos. A maioria (57\%, n=97) não era procedente de Curitiba. 
Tabela 1 - Características dos pacientes ( $n=168$ )

\begin{tabular}{|c|c|c|}
\hline \multicolumn{2}{|c|}{ Características dos pacientes } & \multirow{2}{*}{$\begin{array}{c}\text { n (\%) } \\
90(53)\end{array}$} \\
\hline Sovo & Masculino & \\
\hline JeגU & Feminino & $78(47)$ \\
\hline \multirow{4}{*}{ Idade (anos) } & $0-2$ & $55(33)$ \\
\hline & $2-5$ & $59(35)$ \\
\hline & $5-10$ & $33(20)$ \\
\hline & $10-18$ & $21(12)$ \\
\hline \multirow{2}{*}{ Procedente de Curitiba } & $\operatorname{Sim}$ & $71(43)$ \\
\hline & Não & 97 (57) \\
\hline
\end{tabular}

Fonte: Elaborada pelos autores (2019).

Cento e quarenta e seis (87\%) referiram que tinham conhecimento de que passariam pela triagem ao chegar para atendimento. Como demonstrado no Gráfico 1, as principais razões para terem escolhido levar a criança para atendimento no PS foram: "Maior resolutividade/confiança no atendimento no hospital" (49\%, n=82); "Realizar acompanhamento clínico no hospital" (12\%, n=21); Ter pediatras/especialistas no prontosocorro (12\%, n=20): "Possibilidade de realizar exames" (7\%, n=13); e "Encaminhado / orientado a vir da Unidade Básica de Saúde (UBS) ou da Unidade de Pronto Atendimento (UPA)" (7\%, n=12).

Gráfico 1 - Razões de procura ao PS

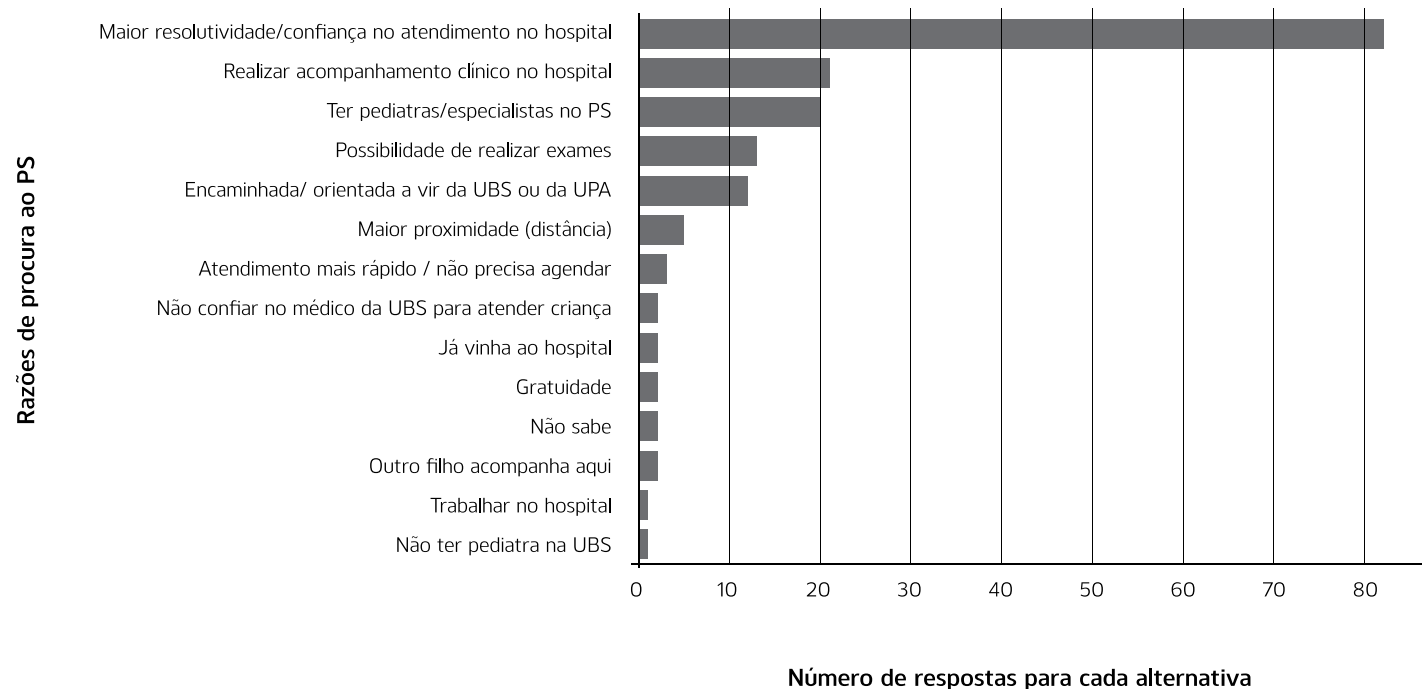

Fonte: Elaborado pelos autores (2019). 
Conforme demonstrado na tabela 2, as principais queixas de saúde relatadas pelos acompanhantes foram, em primeiro lugar, febre (26\%), seguida de tosse (13\%) e vômitos (10\%).Quando questionados se achavam que o problema de saúde atual da criança era uma urgência, $68 \%$ responderam que sim, enquanto 29\% não achavam ser uma urgência e 3\% não sabiam.

Dentre os acompanhantes que consideraram o quadro de saúde atual como uma urgência, as principais queixas de saúde apresentadas foram febre (27\%); vômitos (11\%); dor abdominal (10\%) e tosse (8\%). Ainda entre os que consideram um quadro urgente, $28 \%$ das crianças tinham menos de 2 anos e os principais motivos para terem procurado o PS para atendimento foram: "Maior resolutividade/confiança no atendimento no hospital" (49\%); "Ter pediatras/especialistas no pronto-socorro" (12\%); e "Realizar acompanhamento clínico no hospital" (9\%).

A maioria (63\%) dos acompanhantes referiu que já havia procurado atendimento prévio pelo mesmo motivo, sendo que $41 \%$ relataram que o local prévio procurado foi a UPA e $22 \%$ a UBS. Desses, $41 \%$ haviam procurado atendimento em até 1 dia antes; $17 \%$ em ate 2 dias antes e 25\% há 5 dias ou mais (tabela 2). Dos 106 participantes que referiram ter procurado atendimento prévio, 52\% relataram não terem ficado satisfeitos com o atendimento recebido nesses locais, sendo as principais razões de insatisfação: "Não concorda com o diagnóstico ou com a conduta" (25\%), "Não ter resolvido o problema" (21\%) e "A criança não foi bem tratada (falta de atenção)" (12\%).

Tabela 2 - Características dos atendimentos.

\begin{tabular}{|c|c|c|}
\hline \multicolumn{2}{|c|}{ Caracteristicas dos atendimentos } & \multirow{2}{*}{$\begin{array}{c}\text { n (\%) } \\
43(26)\end{array}$} \\
\hline \multirow{7}{*}{ Principais queixas $(n=168)$} & Febre & \\
\hline & Tosse & $22(13)$ \\
\hline & Vômitos & $16(10)$ \\
\hline & Dor abdominal & $15(9)$ \\
\hline & Manchas na pele & $12(7)$ \\
\hline & Dor de cabeça & $11(6)$ \\
\hline & Outros & $49(29)$ \\
\hline \multirow{3}{*}{ Percepção de urgência (n=168) } & Urgência & $115(68)$ \\
\hline & Não urgência & $48(29)$ \\
\hline & Não sabe & $5(3)$ \\
\hline \multirow{2}{*}{ Procura prévia por atendimento $(n=168)$} & $\operatorname{Sim}$ & $106(63)$ \\
\hline & Não & $62(37)$ \\
\hline \multirow{5}{*}{ Há quantos dias houve a procura $(n=106)$} & 1 & $44(41)$ \\
\hline & 2 & $18(17)$ \\
\hline & 3 & $13(12)$ \\
\hline & 4 & $5(5)$ \\
\hline & $\geq 5$ & $26(25)$ \\
\hline \multirow{6}{*}{ Local da procura prévia $(n=106)$} & UPA & $44(41)$ \\
\hline & UBS & $23(22)$ \\
\hline & PS (outro hospital) & $16(15)$ \\
\hline & PS HPP & $15(14)$ \\
\hline & Pediatra & $7(7)$ \\
\hline & Odontologista & $1(1)$ \\
\hline \multirow{2}{*}{ Satisfeito com o atendimento prévio $(n=106)$} & $\operatorname{Sim}$ & $51(48)$ \\
\hline & Não & $55(52)$ \\
\hline
\end{tabular}

Fonte: Elaborada pelos autores (2019). 
Dentre os que procuraram a UPA como atendimento prévio, 70\% relataram não terem ficado satisfeitos com o atendimento, sendo as principais queixas: "Não concorda com o diagnóstico ou com a conduta" (20\%). "Não ter resolvido o problema" (18\%) e "A criança não foi bem tratada (falta de atenção)" (14\%). Já dos que procuraram a UBS como atendimento prévio, 61\% relataram não terem ficado satisfeitos com o atendimento, tendo como principais motivos diferentes dos demais: "Não conseguiu atendimento médico" (29\%), "Não havia médico no local" (21\%) e "Não concorda com o diagnóstico ou com a conduta" (21\%).

Cento e nove acompanhantes (65\%) referiram que levaram o paciente ao PS outras vezes nos últimos 6 meses devido a outras queixas. Dentre esses, 25\% disseram que levaram até 1 vez; 24\% até 2 vezes; 23\% mais que 5 vezes. A maioria dos acompanhantes (65\%) relatou que a criança frequenta ou já frequentou a UBS. Noventa e cinco (56\%) entrevistados disseram que a criança realiza consultas de puericultura de rotina. Quando questionados se já haviam recebido alguma orientação por profissionais da saúde sobre percepção de sinais de alarme na criança, 107 (63\%) disseram que nunca receberam nenhuma orientação prévia. Quando questionados se algum profissional da área da saúde já havia Ihes orientado sobre quais as diferenças para procura de atendimento entre a UBS, UPAS e hospitais, 139 (83\%) referiram não ter recebido em nenhum momento essa informação.

\section{DISCUSSÃO}

Nos meses em que foi realizada a coleta de dados, $66 \%$ das consultas triadas foram classificadas como não urgentes, valor ligeiramente superior aos 37 a $60 \%$ encontrados nos Estados Unidos ${ }^{17}$, também aos 40 a $60 \%$ em estudos europeus ${ }^{5.7}$ e $59 \%$ em um estudo argentino ${ }^{18}$. Entretanto, o percentual encontrado no hospital em questão pode ser considerado baixo quando analisado frente aos demais dados brasileiros de consultas não urgentes em PS pediátricos, em que são demonstrados valores entre 56 e 96\% de consultas não urgentes nessas unidades $2 ., 613$.

A febre foi citada como principal preocupação quando questionados pela queixa de saúde, representando $25 \%$ de todas as queixas. Quando em vista, outros estudos sobre consultas não urgentes nos PS, a febre manteve-se unanimemente como principal queixa ${ }^{6.1019}$. O termo "febrefobia" foi cunhado em $1980^{20}$ para representar a ansiedade e insegurança por parte dos pais frente a um quadro de febre, com consequentes especulações inapropriadas sobre febre, como possibilidades de sequelas graves (principalmente neurológicas) e possibilidade de elevação da temperatura até altos níveis, caso não tratada com antitérmicos. Outros autore $^{21}$ afirmaram que os pais acreditam que a febre signifique mais uma doença do que um sinal ou sintoma de uma enfermidade, levando a um erro de entendimento do processo de doença. Somando-se a isso, também já foi verificado que, quando o sintoma preocupante era febre, os pais apresentavam mais dificuldade em diferenciar o grau de urgência, tendendo a aumentar a visão de gravidade ${ }^{22}$.

A razão de procura mais citada pelos acompanhantes foi "maior resolutividade/confiança no atendimento no hospital". Um estudo em Recife em cinco PS pediátricos, encontrou que a legitimidade do PS perante a população ("é uma boa referência", "o atendimento é o melhor", "tem mais recursos", "muito conceituado") também foi o motivo de justificativa de procura mais citado 2 . Ademais, em Belo Horizonte ${ }^{6}$, outro estudo reafirmou tal preferência aos serviços de PS a partir de depoimentos de mães que relataram como motivos principais de busca ao PS: resolutividade, qualidade, especificidade para atendimento de crianças, facilidade de acesso, experiências e recomendações. Não obstante a essas razões já corroboradas, o hospital estudado em questão possui características próprias que podem contribuir para essa conduta dos responsáveis - é o 
único hospital exclusivamente pediátrico que recebe pacientes do SUS em Curitiba e região metropolitana, com importante renome e reputação na comunidade. Isso pode representar uma referência clara para os pais quando pensam em cuidados pediátricos.

A segunda principal razão citada pelos responsáveis foi a da procura pelo serviço porque a criança já realiza acompanhamento clínico no hospital. As queixas agudas apresentadas para a procura ao PS não tinham relação com as comorbidades prévias do paciente pois, caso tivessem, os pacientes seriam priorizados e, dessa forma, nem participariam do estudo. Assim, o julgamento equivocado das razões de procura ao PS estarem relacionadas à comorbidade ou não, pode significar dificuldade de entendimento dos pais ou também alguma deficiência no fornecimento das informações pelos profissionais de saúde. Isso pode acarretar um acúmulo de funções ao hospital, uma vez que ele acaba por absorver todas as queixas das crianças atendidas e também trazer algum prejuízo às crianças, que possivelmente não possuem um pediatra de acompanhamento de puericultura e acabam recorrendo a consultas apenas com especialistas.

Em terceiro lugar, a razão mais citada foi o fato do serviço do PS contar com pediatras/especialistas para atendimento. Outros autores ${ }^{11}$ também demonstraram que os pais possuem maior confiança no serviço de PS por possuírem pediatras e serem locais mais capacitados a atender crianças. Tal preferência pelo PS também foi demonstrada em um trabalho qualitativo, em que os pais demonstraram claramente que acreditam que os médicos do PS pediátrico são mais eficientes e mais qualificados para lidar com doenças agudas $^{10}$.

Por fim, a possibilidade de realização de exames no PS apresentou-se como a quarta principal razão de procura. Um estudo português verificou que, apesar da benignidade da maioria das doenças atendidas no PS pediátrico avaliado, realizaram-se exames complementares em 31,3\% dos casos. Com isso, os autores afirmaram que a superutilização dos exames complementares, comparativamente aos diagnósticos não urgentes e a utilização de técnicas terapêuticas, poderá continuar a condicionar a população ${ }^{23}$. Isso reforça a prática de recorrer primeiramente aos PS e justifica a manutenção desta controvérsia, além de dificultar cada vez mais a procura às unidades de menor complexidade, que poderiam proporcionar atendimento e solucionar grande parte dos problemas não urgentes. Assim, os médicos assistentes devem ter a responsabilidade de solicitar exames com a parcimônia necessária e se responsabilizar por educar a população em relação a essa questão.

Apesar da proximidade física do PS ter sido demonstrado como um fator predisponente às consultas não urgentes $^{18}$, no atual estudo a razão de distância foi apenas a sexta mais citada. Além disso, ao analisar a procedência das crianças, 57\% delas não residiam em Curitiba. Ou seja, a maioria dos entrevistados era proveniente de outros municípios e, mesmo por questões não urgentes, optaram por procurar o PS pediátrico. Dessa forma, pode-se supor que a procura está fortemente inerente à principal razão já citada, de confiança no serviço, demonstrando que muitas vezes os responsáveis ainda optarão pelo serviço de reconhecida qualidade independentemente da distância.

A visão de urgência que os responsáveis têm do quadro de saúde da criança representa um importante fator no momento da escolha entre levar a criança ao PS ou a outro serviço ${ }^{9.24}$. Além disso, usualmente o entendimento da gravidade de um quadro de saúde agudo pelos responsáveis é superestimado ${ }^{11,24,25}$, tal como demonstrado no presente estudo, em que 68\% dos cuidadores referiram entender como urgente o quadro de saúde atual da criança. Por outro lado, é imprescindível considerar os diferentes entendimentos possíveis do que significa uma urgência ou uma emergência. Ao estudar perspectivas de urgências e emergências de profissionais de saúde e de usuários, uma autora ${ }^{26}$ trouxe à tona que existem diferentes entendimentos para tais conceitos. Por um lado, há a visão médica e técnica desses conceitos, com definições "singularizantes" 
e "automatizadas" em relação às outras esferas da vida que não a biológica. Por outro lado, há a definição social dos que não possuem o conhecimento técnico, porém têm a carga cultural e social embutida, que determinarão sua própria classificação de uma urgência, configurando basicamente o que pode pôr a sua vida em risco. Dessa forma, muitas vezes há o desencontro entre entendimentos de especialistas e usuários e, assim, a autora conclui que os entendimentos de urgência são plurais e devem ser analisados nos seus diversos contextos e participantes ${ }^{26}$. As discrepâncias de visão de urgência entre profissionais e usuários, tal como demonstrado no atual estudo, demonstra uma grande dificuldade de padronização. Consequentemente, uma análise completa com os diferentes pontos de vista deve ser realizada para entendimento da procura com a devida contextualização de cenários e circunstâncias.

A deficiência de resolutividade e de acesso aos serviços de atenção à saúde, sem adequada referência e contrarreferência entre os serviços e articulação entre as redes de cuidados, representam um caminho de peregrinação dos usuários do SUS ${ }^{27}$. Dentre os 106 acompanhantes que disseram ter procurado auxílio prévio em outros serviços, os locais mais buscados foram as UPAS em primeiro lugar (41\%), seguidos das UBS (22\%). Tal resultado demonstra que grande parte dos usuários ainda buscou auxilio nos níveis de atenção primária e secundária antes de recorrer ao PS. O pressuposto pelo SUS é que haja UBS e UPAS como os locais mais próximos às pessoas, com cobertura populacional abrangente e alta resolutividade ${ }^{28}$. Entretanto, sabe-se que na realidade atual, a escassez tanto de estrutura física quanto de pessoal, principalmente nos níveis de atenção primária e secundária é uma questão presente e que gera dificuldades de acesso à população ${ }^{29}$. Dessa forma, esperar uma procura escalonada aos serviços de saúde pelos usuários, sendo que a resolutividade dos serviços não ocorre como deveria, por vezes não é algo razoável.

A maioria dos que buscaram auxílio em outros locais previamente relatou ter ficado insatisfeita com o atendimento por diferentes razões. Enquanto os que haviam procurado previamente a UPA como apoio, queixaram-se de questões relativas ao quadro de saúde (insatisfação por discordar com o diagnóstico ou com a conduta tomada pelo médico, ou que a conduta tomada não foi resolutiva), quem havia procurado a UBS queixou-se principalmente de problemas estruturais (não ter conseguido atendimento médico, não haver médico no local).

O que fica claro no presente estudo é a oposição entre o principal fator de insatisfação em relação aos outros serviços procurados previamente e a principal razão citada para procura ao PS. Ou seja, reforçase como principal aspecto valorizado pelos cuidadores a confiança e a crença de resolutividade no serviço (no hospital) e que estes estão mais claramente relacionados aos serviços de atenção terciária. Um estudo brasileiro $^{30}$ reforça tal conceito, demonstrando que a acessibilidade, a confiabilidade e a resolutividade foram os fatores essenciais que os usuários levaram em conta para a escolha dos serviços. Além disso, demonstrou que tais fatores têm pendido mais à procura por PS, não por que os PS e os hospitais sejam locais ideais, mas pela imagem denegrida das UBS e UPAS. Este conceito também é reiterado por outro autor ${ }^{31}$, o qual explicita que há um conceito equivocado de complexidade que leva a população a subvalorizar a atenção primária por considerar que ela seja menos preparada tecnologicamente.

Outra possibilidade que pode ser refletida para o entendimento dos principais motivos de insatisfação dos cuidadores é a análise conjunta do tempo em que ocorreu a procura prévia. Quarenta e dois por cento alegaram ter ido a um serviço em até 24 horas antes de estarem no PS, o que representa um tempo curto de reavaliação. Uma possivel suposição seria o fato de que os pais não tenham sido devidamente orientados em relação à evolução da doença, uso de medicações e acompanhamento do quadro com atenção aos sinais de alarme, além de devidas orientações para procurar novamente o serviço, se necessário. Porém, também há a possibilidade de ansiedade dos pais e preocupação exacerbada em relação ao quadro de saúde, com reforço 
de tal hipótese ao considerarmos a visão de urgência referida pelos pais (68\% consideraram que o quadro atual da criança seria uma urgência).

Mais da metade (56\%) dos cuidadores relatou que a criança realizava consultas de rotina, sendo existentes na literatura alguns fatores nessas consultas que podem ser determinantes para a ida ou não ao PS. Primeiramente, uma das características é a presença de um atendimento de atenção primária de "alta qualidade", que é principalmente mensurado nos estudos por: "prestadores de cuidados primários que respeitam o que os pais têm a dizer", "que ouvem atentamente", "que passam tempo suficiente com as crianças", refletido principalmente nas visitas entre os menores de 2 anos ${ }^{17,32}$. Também já foi demonstrado como fator determinante a continuidade nas consultas de puericultura, caracterizada por um maior número de consultas com o mesmo médico, e com isso mostrou-se que pacientes de baixa continuidade apresentaram um aumento de risco de utilizarem o PS e de serem hospitalizados ${ }^{33}$. Ou seja, pode-se entender que o fato de ter consultas ou ter acesso à atenção primária varia como fator de procura ao PS conforme a avaliação de outras variáveis, como qualidade e frequência.

Na questão de educação em saúde, foi demonstrado que na maior parte dos casos os cuidadores referiram não ter recebido informações, especialmente em relação às diferenças entre os níveis de atenção. Alguns estudos já demonstraram que pais com menor educação em saúde, além de superestimarem os sintomas e a gravidade dos filhos, procuram atendimento imediato mais precocemente ${ }^{24,27.34}$. Em contrapartida, cuidadores com mais conhecimento relataram buscar segurança para o gerenciamento contínuo da doença e valorizaram os relacionamentos próximos com seu médico, além de estarem dispostos a esperar mais tempo por uma consulta e terem mais segurança de iniciar algum cuidado em casa.

A transmissão da educação em saúde é fortemente dependente dos profissionais de saúde atuantes e da sua colaboração nessa função, pois eles representam as principais fontes de informação perante a população. Com foco à área médica, pode-se avaliar a formação acadêmica brasileira, que tem em seu histórico eixos norteadores que foram principalmente centrados de modo tecnocientífico e sob a ótica estritamente dos processos patológicos, tendo sido pouco explorado o aprendizado para a realidade social em que são inseridos na prática médica ${ }^{35}$. Atualmente, os cursos de medicina passam por um processo de adaptação de currículo com direcionamento para a conexão da formação médica com os serviços públicos de saúde, em busca de que as práticas de saúde sejam norteadas na produção da integralidade e da humanização do cuidado ${ }^{36}$. Pode-se esperar, com base em tais mudanças curriculares, um novo olhar gerado sobre o sujeito como indivíduo e suas complexidades sociais.

A partir do atual estudo e com base em resultados já alcançados em pesquisas prévias, sugere-se a concentração de esforços e investimentos em dois principais campos como propostas de resolução às visitas não urgentes aos PS, o do acesso à atenção básica e o da educação em saúde aos responsáveis. Primeiramente. melhorar o acesso aos serviços de atenção básica e incentivar a puericultura com adequada continuidade dos cuidados das crianças, com a construção de um relacionamento médico-paciente de confiança, além de proporcionar melhor atenção à criança ${ }^{10,1220}$. Por fim, prover um sistema adequado de acolhimento nas UBS e possibilitar marcação de consultas no mesmo dia para queixas agudas, além de melhorar a comunicação da equipe de saúde com os pacientes e prolongar o horário de expediente são algumas sugestões para melhorar o acesso à atenção primária10 ${ }^{10}$. A segunda medida que é também proposta por alguns autores com resultados expressivos é a melhora da educação em saúde dos responsáveis. Os pais desejam receber informação e a melhora da compreensão sobre doença aguda, gravidade da doença, onde e quando procurar atendimento pode levar a uma redução no uso não urgente dos PS ${ }^{10.22 .37}$. 


\section{CONCLUSÃO}

O principal aspecto valorizado pelos cuidadores foi a confiança e a crença de resolutividade no hospital, que está claramente relacionada aos serviços de atenção terciária. Procurar entender a visão do usuário do sistema é fundamental para a compreensão dos motivos e de como ocorre a procura por serviços de saúde. Sem entender a vulnerabilidade da população, seus medos, suas crenças e seu caminho percorrido em busca de auxílio, é provável que suas condutas sejam julgadas erroneamente, avaliando apenas o ponto final, a chegada "inadequada" ao PS.

Além disso, foi demonstrada a importância da população ter acesso à saúde básica e à educação como fatores para reduzir as visitas não urgentes ao PS. Provavelmente, enquanto áreas de saúde estiverem descobertas de médicos, faltarem senhas para o atendimento de acolhimento, faltarem medicações nas UBS, os usuários procurarão resolver seus anseios e suas preocupações à sua maneira, procurando a porta de entrada ao seu alcance, até que seja atendido e acolhido.

Por fim, pretende-se demonstrar à comunidade de pediatras e médicos em geral as angústias e anseios dos pais ao apresentar uma visão "do outro lado da mesa de atendimento", apresentando as perspectivas dos usuários. Por vezes que essa visão não seja compartilhada pelos profissionais de saúde, propõe-se assim que haja uma visão empática de todo processo de doença e de procura por auxílio que ocorre pelos cuidadores.

O estudo apresentou algumas limitações. Por ter sido um protocolo extenso e aplicado por meio de um entrevistador, consequentemente obteve-se uma amostra reduzida e selecionada por conveniência, além de tempo limitado. Também por se tratar de um pronto-socorro de uma unidade hospitalar, pode representar uma limitação à análise com outras unidades. Deve-se levar em consideração ainda, como limitação já citada previamente também em outros estudos da área, a classificação utilizada para caracterizar os não urgentes ser proveniente da própria instituição, podendo limitar maiores comparações.

Para o futuro, propõe-se uma ampliação do estudo, com aplicação de questionário aberto para as principais razões de procura, buscando uma análise qualitativa e mais aprofundada da questão.

\section{REFERÊNCIAS}

1. Conselho Federal de Medicina. Resolução n 2077/14 de 24 de julho de 2014, Brasil. Dispõe sobre a normatização do funcionamento dos Serviços Hospitalares de Urgência e Emergência, bem como do dimensionamento da equipe médica e do sistema de trabalho.

2. Kovacs MH, Feliciano KVO, Sarinho SW, Veras AACA. Acessibilidade às ações básicas entre crianças atendidas em serviços de prontosocorro. J Pediatr. 2005;81(3):251-258.

3. Caccia-Bava MC, Pereira MJB, Rocha JSY, Martine EZ. Pronto atendimento ou atenção básica: escolhas dos pacientes no SUS. Medicina (Ribeirão Preto On-line) 2011;44(4):347-54.

4. Sturm JJ, Hirsh D. Weselman B, Simon HK. Reconnecting patients with their primary care provider: an intervention for reducing nonurgent pediatric emergency department visits. Clin Pediatr (Phila). 2014:53(10):988 -994.

5. Wong AC, Claudet I, Sorum P. Mullet E. Why do parents bring their children to the emergency department? A systematic inventory of motives. Int J Family Med. 2015;2015:10p.

6. Rati RMS, Goulart LMHF, Alvim CG, Mota JAC. "Criança não pode esperar": a busca de serviço de urgência e emergência por mães e suas crianças em condições não urgentes. Cien Saude Colet. 2013:18(12):3663-3672.

7. Vedovetto A, Soriani N. Merlo E, Gregori D. The burden of inappropriate emergency department pediatric visits: why Italy needs an urgent reform. Health Serv Res. 2014:49(4):1290-1305.

8. Rafael MS, Portela SL, Sousa P. Fernandes AC. Utilização do serviço de urgência pediátrica: a experiência de um centro português. Sci 
Med. 2017:27(1):1-8

9. Phelps K, Taylor C, Kimmel S, Nagel R, Klein W, Puczynski S. Factors associated with emergency department utilization for nonurgent pediatric problems. Arch Fam Med. 2000;9:1086-1092.

10. Berry A, Brousseau D, Brotanek J M. Tomany-Korman S. Flores G. Why do parents bring children to the emergency department for nonurgent conditions? A qualitative study. Ambul Pediatr. 2008;8(6):360-367.

11. Kubicek K, Liu D, Beaudin C, Supan J, Weiss G, Lu Y, et al. A profile of nonurgent emergency department use in an urban pediatric hospital. Pediatr Emerg Care. 2012;28(10):977-984.

12. Fieldston ES, Alpern ER, Nadel FM, Shea JA, Alessandrini EA. A qualitative assessment of reasons for nonurgent visits to the emergency department - Parent and health professional opinions. Pediatr Emer Care. 2012:28(3):220-225.

13. Arrué AM, Neves ET, Buboltz FL, Jantsch LB, Zanon BP. Demanda de um pronto-socorro pediátrico: caracterização dos atendimentos de enfermagem. Rev enferm UFPE on-line. 2013;7(4):1090-1097.

14. Oliveira, TMV. Amostragem não probabilística: adequação de situações para uso e limitações de amostras por conveniência, julgamento e quotas. Rev Administração On-line [periódico na internet]. 2001 - Jul-Set [acessado 2018 Set]; 2(3): [cerca de 16p.]. Disponível em:https:// www.fecap.br/adm_online/art23/tania2.htm

15. Marotti J, Galhardo APM, Furuyama RJ, Pigozzo MN, Campos TN, Laganá DC. Amostragem em pesquisa clínica: tamanho da amostra. Rev Odontol Univ São Paulo. 2008:20(2):186-194.

16. Carmo H, Ferreira MM. Amostragens não probabilísticas. In: Carmo H. Ferreira MM. Metodologia da Investigação: Guia para autoaprendizagem. 2. ed. Lisboa: Universidade Aberta. 2008;p.215-219.

17. Brousseau DC, Hoffmann RG, Nattinger AB, Flores G, Zhang Y, Gorelick M. Quality of primary care and subsequent pediatric emergency department utilization. Pediatrics. 2007:119(6):1131-1138.

18. Vinelli NF, Mannucci C, Laba NI, Del Vecchio L, Valerio A, Lago MI, et al. Consultas no urgentes al departamento de urgencias de un hospital pediátrico. Arch Argent Pediatr. 2011:109(1):8-13.

19. Benahmed N, Laokri S, ZhangWH, Verhaeghe N, Trybou J, Cohen L, et al. Determinants of nonurgent use of the emergency department for pediatric patients in 12 hospitals in Belgium. Eur J Pediatr. 2012:171(12):1829-1837.

20. Schmitt BD. Fever phobia: misconceptions of parents about Fevers. Am J Dis Child. 1980;134(2):176- 181.

21. Crocetti M, Moghbeli N, Serwint J. Fever phobia revisited: have parental misconceptions about fever changed in 20 years? Pediatrics. 2001;107(6):1241-1246.

22. May M, Brousseau DC, Nelson DA, Flynn KE, Wolf MS, Lepley B, et al. Why parents seek care for acute illness in the clinic or the ed: the role of health literacy. Acad Pediatr. 2018:18(3):289-296.

23. Oliveira A, Guerra MP, Peralta L, Almeida S, Cunha Fl, Bicho A. Serviço de urgência pediátrico: casuística de um hospital com serviço de pediatria geral. Saúde Infantil. 2010;32(2):53-58.

24. Kalidindi S, Mahajan P. Thomas R, Sethuraman U. Parental perception of urgency of illness. Pediatr Emerg Care. 2010:26(8):549-553.

25. Freitas AC, Moreira AR, Tomé S, Cardoso R. Motivos de recurso ao serviço de urgência pediátrica.Nascer e Crescer. 2016;25(3):136-140.

26. Jacquemot AC. Urgências e emergências em saúde: perspectivas de profissionais e usuários. Rio de Janeiro: Fiocruz. 2005.

27. Buboltz FL. Ações de cuidado da família à criança atendida em pronto-socorro pediátrico a partir do seu universo sociocultural [dissertação]. Santa Maria (RS): Universidade Federal de Santa Maria. 2013.

28. Ministério da Saúde (MS). Política Nacional de Atenção Básica. Brasília: MS. 2012.

29. Souza ECF, Vilar RLA, Rocha NSPD, Uchoa AC, Rocha PM. Acesso e acolhimento na atenção básica: uma análise da percepção dos usuários e profissionais de saúde. Cad Saude Publica. 2008:24(1):100-110.

30. Oliveira LH, Mattos RA, Souza AIS. Cidadãos Peregrinos: os 'usuários' do SUS e os significados de sua demanda a pronto-socorros e hospitais no contexto de um processo de reorientação do modelo assistencial. Cien Saude Colet. 2009:14(5):1929-1938.

31. Mendes EV. As redes de atenção à saúde. Brasília: Organização Pan-americana da Saúde. 2011.

32. Alele FO, Emeto TI, Callander EJ, Watt K. Non-urgent paediatric emergency department presentation: a systematic review. J Paediatr Child Health. 2018;55(3): 271-277.

33. Christakis DA, Mell L, Koepsell TD, Zimmerman FJ, Connell FA. Association of lower continuity of care with greater risk of emergency department use and hospitalization in children. Pediatrics. 2001:103(3):524-529.

34. Yoffe SJ, Moore RW, Gibson JO, Dadfar NM, McKay RL, McClellan DA, et al. A reduction in emergency department use by children from 
a parent educational intervention. Fam Med. 2011;43(2):106-111.

35. Machado CDB, Wuo A, Heinzle M. Educação médica no Brasil: uma análise histórica sobre a formação acadêmica e pedagógica. Rev Bras Educ Med. 2018;42(4):66-73.

36. Nogueira MI. As mudanças na educação médica brasileira em perspectiva: reflexões sobre a emergência de um novo estilo de pensamento. Rev Bras Educ Med. 2009:33(2):262-270.

37. McWilliams DB, Jacobson RM, Van Houten HK, Naessens JM, Ytterberg KL. A program of anticipatory guidance for the prevention of emergency department visits for ear pain. Arch Pediatr Adolesc Med. 2008:162(2):151-156. 